\title{
Alterstice
}

Revue internationale de la recherche interculturelle

International Journal of Intercultural Research

Revista International de la Investigacion Intercultural

\section{Points de vue d'élèves issus de l'immigration sur leur expérience socioscolaire en contexte montréalais défavorisé}

\section{Fasal Kanouté, Justine Gosselin-Gagné, Rajae Guennouni Hassani et Christine Girard}

Volume 6, numéro 1, 2016

Prendre en compte la diversité à l'école

URI : https://id.erudit.org/iderudit/1038275ar

DOI : https://doi.org/10.7202/1038275ar

Aller au sommaire du numéro

Éditeur(s)

Alterstice

ISSN

1923-919X (numérique)

Découvrir la revue

Citer cet article

Kanouté, F., Gosselin-Gagné, J., Guennouni Hassani, R. \& Girard, C. (2016).

Points de vue d'élèves issus de l'immigration sur leur expérience socioscolaire en contexte montréalais défavorisé. Alterstice, 6(1), 13-25.

https://doi.org/10.7202/1038275ar
Résumé de l'article

Notre étude porte sur les regards croisés de différents protagonistes autour de l'expérience scolaire d'élèves issus de l'immigration dans des environnements scolaires qui conjuguent immigration récente et défavorisation. Cet article partage les données relatives au discours des élèves. Ces derniers témoignent de manière complexe de leur identification ethnoculturelle et de leur appréhension générale de l'altérité ethnoculturelle dans leur réseau des pairs. Ils soulignent les défis rencontrés dans l'appropriation du français mais aussi d'autres facteurs qui constituent des tuteurs de résilience scolaire, notamment l'utilisation des ressources mobilisées par les organismes communautaires.
Droits d'auteur @ Fasal Kanouté, Justine Gosselin-Gagné, Rajae Guennouni Hass et Christine Girard, 2016
Cedocument est protége par la loi sur le droit d'auteur. L'utilisation des services d'Érudit (y compris la reproduction) est assujettie à sa politique d'utilisation que vous pouvez consulter en ligne. 


\section{7}

alterstice

Revue Internationale

de la Recherche Interculturelle

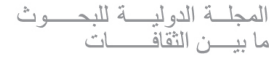

Revista International

de la Investigacion Intercultural

ARTICLE THÉMATIQUE

\section{Points de vue d'élèves issus de l'immigration sur leur expérience socioscolaire en contexte montréalais défavorisé}

Fasal Kanouté ${ }^{1}$, Justine Gosselin-Gagné ${ }^{1}$, Rajae Guennouni Hassani ${ }^{1}$ et Christine Girard ${ }^{1}$

\section{Résumé}

Notre étude porte sur les regards croisés de différents protagonistes autour de l'expérience scolaire d'élèves issus de l'immigration dans des environnements scolaires qui conjuguent immigration récente et défavorisation. Cet article partage les données relatives au discours des élèves. Ces derniers témoignent de manière complexe de leur identification ethnoculturelle et de leur appréhension générale de l'altérité ethnoculturelle dans leur réseau des pairs. Ils soulignent les défis rencontrés dans l'appropriation du français mais aussi d'autres facteurs qui constituent des tuteurs de résilience scolaire, notamment l'utilisation des ressources mobilisées par les organismes communautaires.

\section{Rattachement des auteures}

${ }^{1}$ Université de Montréal, Montréal, Canada

\section{Correspondance}

fasal.kanoute@umontreal.ca

\section{Mots clés}

élèves, immigration, défavorisation, expérience socioscolaire, intégration

\section{Pour citer cet article}

Kanouté, F., Gosselin-Gagné, J., Guennouni Hassani, R. et Girard, C. (2016). Points de vue d'élèves issus de l’immigration sur leur expérience socioscolaire en contexte montréalais défavorisé. Alterstice, 6(1), 13-25. 


\section{Introduction}

Selon le ministère de l'Immigration, de la Diversité et de I'Inclusion (MIDI, 2015), le Québec a accueilli en 2014 50275 immigrants. Parmi les immigrants admis entre 2004 et 2013, 59,3 \% résident, en janvier 2015, dans la région administrative de Montréal. Même si la diversité socioculturelle et ethnique de la société est une donnée structurelle qui n'est pas seulement le fruit de l'immigration récente, cette dernière y contribue par l'hétérogénéité de plus en plus grande de la provenance des immigrants.

L'école étant la plus importante institution à accueillir les enfants et les jeunes de la société, elle reflète cette diversité socioculturelle et ethnique, notamment sur l'île de Montréal. Le Comité de gestion de la taxe scolaire de l'île de Montréal (CGTSIM) quantifie par exemple la présence des élèves dits " issus de l'immigration récente » en définissant trois catégories. Ainsi, à la rentrée scolaire 2014, près de $62 \%$ des élèves de l'enseignement primaire et secondaire du réseau scolaire public de l'île de Montréal sont des élèves issus de l'immigration récente : $22,4 \%$ de la population scolaire est constituée d'élèves de première génération, nés à l'étranger de parents nés à l'étranger, 28,9 \% d'élèves nés au Québec de parents nés à l'étranger et 10,9 \% d'élèves nés au Québec avec un seul parent né à l'étranger (CGTSIM, 2015). On peut légitimement se questionner les critères qui balisent la définition d'un profil aussi hétérogène que celui élèves issus de l'immigration récente, car il est loin d'être évident, de manière générale, de mesurer la présence des minorités ethnoculturelles (Landes, 2011), qu'elles soient issues ou non de l'immigration. Les élèves immigrants de première génération, auxquels nous nous intéressons ici, représentent près d'un élève sur 4 dans les trois commissions scolaires francophones de Montréal, et leurs trois principaux pays de naissance sont l'Algérie, Haïti et le Maroc. Un constat perdure au fil des ans à travers le "portrait socioculturel " des écoles que le CGTSIM fait chaque année: plus l'immigration familiale est récente, plus les élèves sont susceptibles de résider dans une zone défavorisée (CGTSIM, 2015). Par exemple, en 2014, 54,1\% des élèves immigrants de première génération résident dans une zone de ce type, ce qui n'est le cas que de $21,3 \%$ des élèves nés au Québec de parents nés au Québec. Dans ce contexte, l'objectif général de notre étude - dont nous ne rendons compte que d'une partie des résultats dans cet article - porte sur les conditions de prise en charge des besoins des élèves issus de l'immigration récente dans des contextes scolaires faisant face aux effets combinés de l'immigration récente et de la défavorisation.

Les données globales de cette étude ont été recueillies en 2010, avec des objectifs spécifiques de recherche quant à la description et à l'analyse :

1. du point de vue de parents et d'élèves sur l'expérience socioscolaire de ces derniers et la prise en charge intégrée de leurs besoins par l'école et la communauté,

2. du point de vue d'acteurs scolaires sur ces besoins et leur prise en charge,

3. du point de vue d'organismes et de personnels non scolaires sur l'arrimage de leur intervention à celle de l'école,

4. de l'articulation des différentes logiques d'intervention autour de cette prise en charge.

Cet article rend uniquement compte du point de vue des élèves. Les autres données de l'étude ont donné lieu à une première publication croisant les regards de parents et d'intervenants communautaires (Kanouté, Charette, André, Rachédi et Rahm, 2014) et à une deuxième portant sur le point de vue d'acteurs scolaires et d'intervenants communautaires (Kanouté, Lavoie, Guennouni et Charette, 2016). Dans la section qui suit, nous fournissons un aperçu de l'actualisation de la problématisation des défis auxquels font face les élèves issus de l'immigration récente et leur famille.

\section{Les enjeux de l'intervention en contexte scolaire pluriethnique et défavorisé}

La défavorisation rend compte de diverses formes de privation matérielle, et son étude intègre également l'analyse de celles-ci en termes d'inégalités sociales, de distribution déséquilibrée des ressources collectives et d'asymétrie de pouvoir (Glennerster, 2002; Kanouté, 2007a). La prévalence du "faible revenu », un autre indicateur qui permet d'appréhender partiellement la défavorisation, est ainsi plus élevée dans les grands centres urbains comme 
Montréal. La précarité est un autre levier de compréhension de la dynamique de la défavorisation. Selon le Troisième rapport national sur l'état de santé de la population du Québec de 2007, ainsi que l'Enquête canadienne sur le bien-être économique de 2013, les effets de la précarité touchent le logement, l'employabilité, le sentiment d'appartenance à son milieu, l'exercice de la parentalité, le (non) rapport aux institutions, un accès de qualité (ou non) à des services essentiels, la satisfaction de divers besoins au quotidien, etc. II va sans dire que les effets induits par un tel contexte affectent l'expérience scolaire de l'élève, d'autant plus que la culture scolaire est souvent décrite comme étant moins sensible non seulement à ces effets, mais aussi aux spécificités qui relèvent d'une certaine différence des milieux défavorisés sans que soit perceptible une quelconque déficience (Millet et Thin, 2005; Vatz Laaroussi, 1996).

Les élèves issus de l'immigration récente et leurs familles sont aussi susceptibles de faire face, en général, à plusieurs défis d'acculturation à la société d'accueil, en particulier à une nouvelle école (Kanouté et Lafortune, 2011 et 2014). L'acculturation est un processus global d'adaptation psychologique et socioculturelle, pour un individu ou un groupe, au contact d'une ou de cultures autres que sa culture de première socialisation (Amin, 2012; Berry, 2005; Kanouté 2002). Il faut préciser que les défis d'acculturation ne se posent pas seulement aux immigrants de première génération : ils peuvent perdurer pour la deuxième génération, voire la troisième génération, notamment à cause d'une dynamique de stigmatisation-discrimination systémique ou d'un choix de profil d'acculturation de " séparation » ou de " marginalisation » (Berry, 2005). Dans l'étude des rapports intergroupes, notamment de la situation des minorités en contexte migratoire, le concept d'identité ethnoculturelle est très utilisé. Selon plusieurs auteurs (Abou, 2000; Martiniello, 1995; Meintel, 1993), cette dernière consiste en une identité collective qui réfère à l'identification à un groupe sur la base d'une communalisation de divers éléments, dont l'agrégat dépend de différents contextes: histoire, ascendance, modèles culturels, langue, religion, territoire, intérêts économicopolitiques, expériences sociales, etc. Selon Amin, Poussin et Martinez (2008), l'auto-identification ethnoculturelle chez des jeunes issus de l'immigration, dans une étude ayant pour contexte la France, fait référence à l'utilisation de « signifiants ethniques, nationaux et religieux propres à la culture d'origine de leurs parents pour se définir » et la prévalence de cette auto-identification serait corrélée au sentiment d'exclusion ressenti.

Les élèves issus de l'immigration récente vivant en contexte de défavorisation subissent les contrecoups d'un cumul de difficultés dans les conditions d'établissement de leur famille au sein de la société d'accueil. Selon Boudarbat et Grenier (2014, p. 177), « les immigrants sont surreprésentés parmi les travailleurs pauvres titulaires d'un diplôme universitaire. La protection qu'offre la scolarité contre le risque de faible revenu est donc moins bonne pour les immigrants que pour les natifs ». Ainsi, les parents immigrants instruits, qui font face au phénomène de déclassement social vécu à leur arrivée et dont plusieurs vivent dans un quartier dit défavorisé, peuvent soit avoir des pratiques de suivi scolaire conformes aux attentes par rapport à leur capital humain, soit se démobiliser à cause de la frustration ressentie dans un cadre général de stress d'acculturation (Chen, Smith et Mustard, 2010). Il y a aussi des parents qui demeurent extrêmement distants vis-à-vis de l'école (Hohl, 1996), car ils sont sous-scolarisés ou ont eu un parcours migratoire hautement traumatique. Pour des parents allophones ne maîtrisant pas la langue d'enseignement, le suivi scolaire de l'enfant et la communication avec l'école peuvent s'avérer difficiles, surtout si cette dernière ne dispose pas de ressources pour faire la médiation avec eux ou est peu disposée à envisager l'utilisation d'autres langues de communication avec les familles (Kanouté, Vatz Laroussi, Rachédi et Tchimou Doffouchi, 2008).

Certains élèves immigrants, maîtrisant suffisamment le français et n'éprouvant pas de difficultés notables d'apprentissage, font quand même face à un certain défi dans le décodage de ce que la forme scolaire québécoise a de spécifique. Certains élèves issus de l'immigration récente sont confrontés à l'épreuve que constitue l'apprentissage accéléré du français, langue d'enseignement dans leur école et langue principale de la vie publique dans la société. On retrouve ces derniers dans les classes d'accueil, du moins à Montréal. Des témoignages d'élèves et d'enseignants de ces classes (Kanouté et coll., 2008) révèlent la nécessité de tenir compte de la déstabilisation identitaire vécue par les élèves et de la frustration à ne pas pouvoir actualiser en français les savoirs scolaires très bien acquis dans une autre langue. II y a également le cas d'élèves immigrants en " situation de retard scolaire ", à cause, entre autres, d'une scolarité interrompue par la violence et par des trajectoires migratoires chaotiques (Armand, Rousseau, Lory et Machouf, 2011). Presque tous les élèves immigrants font face à un certain deuil d'un ensemble de repères humains, physiques et symboliques (Papazian-Zohrabian, 2013). 
Les relations interethniques à l'école en général, ou plus spécifiquement les interactions sociales entre pairs en contexte scolaire pluriethnique, ont fait l'objet de plusieurs études. Lafortune et Kanouté (2007) ont illustré la situation des élèves d'origine haïtienne, dont plusieurs font l'expérience dans leurs parcours scolaires d'écueils divers résultant d'une action systémique institutionnelle qui les discrimine : procédure d'évaluation injuste ou prévalence de préjugés de la part des acteurs scolaires. Duong et Kanouté (2007), utilisant l'approche sociométrique, ont documenté la " clanisation » du réseau relationnel dans des classes pluriethniques à Montréal. Selon ces auteures, s'il n'y a pas lieu de vouloir systématiquement démanteler les « clans » qui se forment en classe selon le sexe ou l'origine ethnoculturelle et linguistique des élèves, il est important que l'enseignant veille à la régulation des interactions sociales afin de faire bénéficier le groupe de la richesse sociocognitive de la mixité (Cohen, 1994). Un important rapport conjoint du Conseil national de l'évaluation du système scolaire de la France (CNESCO) et du Conseil supérieur de l'éducation du Québec (CSE) a traité de la question générale de la mixité sociale à l'école. Une de ses recommandations porte spécifiquement sur la nécessité d' " améliorer les relations intergroupes dans les classes et les établissements » (CNESCO-CSE, 2015, p. 60). Nous pensons qu'il y a un effet protecteur à ce que l'élève ne soit pas toujours à l'école dans un réseau relationnel monoethnique.

Les défis que nous venons de présenter sont plus complexes dans les grands centres urbains comme Montréal, où s'installent la majorité des familles immigrantes. Dans ces contextes, les écoles ne peuvent se passer de la collaboration avec les ressources des communautés d'appartenance des élèves et de leurs familles, ou du moins de prendre acte de leur fréquentation par ces derniers. II n'y a pas de définition univoque de "communauté ", mais nous pouvons les définir comme des "territoires de proximité " (Dansereau et Germain, 2002; Vallet, 2005), physiques ou virtuels arpentés par les individus et groupes sociaux à la recherche de ressources coordonnées à leurs besoins divers. Selon Lareau (2003), les ressources communautaires sont nécessaires pour compléter la socialisation de l'enfant, surtout si la famille vit un cumul de vulnérabilités sociales, dû à la précarité ou aux effets de l'immigration récente. Par exemple, les activités ludiques ou sportives auxquelles sont inscrits les enfants leur font vivre des interactions sociales diversifiées avec d'autres adultes "significatifs", ce qui leur permet de se socialiser stratégiquement aux codes sociaux « qui comptent » pour se faire une place dans la société.

Au Québec, l'organisme communautaire est une ressource emblématique de cette communauté, avec des objectifs d'aide à l'actualisation du capital humain et social des familles (alimentation, logement, recherche d'emploi), à l'émergence d'une citoyenneté inclusive dans les quartiers (Ninacs, 2008; Vatz Laaroussi, Kanouté et Rachédi, 2008), à l'amélioration de la situation socioscolaire des jeunes (Kanouté et coll., 2014). Pour l'élève immigrant, le milieu communautaire est une ressource complémentaire pour fixer les apprentissages scolaires, mais aussi un espace de socialisation pour se retrouver et apprivoiser la société d’accueil (Kanouté et coll., 2014; Rahm, 2007).

\section{Approche méthodologique}

Après cette synthèse actualisée de la recension des écrits qui a inspiré notre étude, nous présentons brièvement notre approche méthodologique, qui a guidé la cueillette et l'analyse de nos données. Nous considérons qu'un environnement scolaire est constitué d'une école et d'un ou de plusieurs organismes communautaires situés sur le territoire qu'elle dessert et dont les services sont utilisés par les élèves et leurs familles. Nous avons opté pour une approche compréhensive et qualitative (Yin, 2003), avec un échantillon non probabiliste, ainsi que pour l'entrevue semi-dirigée comme outil de collecte de données. Les trois environnements scolaires ciblés (M1, M3, M9) sont autour d'écoles primaires francophones conjuguant défavorisation et présence significative de familles issues de l'immigration.

Même si nous avons au total rencontré 31 duos parent-élève, un élève seul, 10 acteurs scolaires et 7 intervenants communautaires, les résultats discutés dans cet article ne concernent que le discours des 32 jeunes (J) des milieux M1 (J1 à J10), M3 (J1 à J13) et M9 (J1 à J8 et J11) ${ }^{1}$. Nous avons obtenu l'autorisation des parents avant de rencontrer leurs enfants. Les entrevues se sont déroulées dans les écoles, chez les familles ou dans les locaux des organismes communautaires, à la convenance des parents. La grille d'entrevue avec les élèves a été construite

\footnotetext{
${ }^{1}$ Les données concernant les jeunes J9 et J10 du milieu M9 ont été supprimées après transcription et codification, à la suite du désistement des parents.
} 
autour des grandes dimensions suivantes : profil sociodémographique et parcours migratoire, vécu socioscolaire et suivi parental et enfin vie de quartier. Les entrevues se sont déroulées en français et ont été enregistrées.

Au moment des entrevues, les 32 élèves (19 filles et 13 garçons) que nous avons rencontrés sont âgés de 8 à 16 ans : 8-9 ans (7), 10-11 ans (11), 12 ans (10), 13-14 ans (3) et 16 ans (1). Ils sont immigrés de première (22) ou de deuxième (10) génération. Ceux de première génération sont nés dans les pays suivants: Haïti (4), Algérie (4), Mexique (3), Tunisie (3), Cameroun (2), Moldavie (2), Colombie (1), île Maurice (1), Nigéria (1), Roumanie (1). Les jeunes qui ont commencé l'école dans leur pays d'origine sont au nombre de 19, dont 16 ont fréquenté la classe d'accueil à leur arrivée dans le système scolaire québécois. Les élèves du primaire sont au nombre de 28 et ceux du secondaire de 4. En effet, même si l'étude a visé des environnements scolaires autour d'écoles primaires, certaines familles ont suggéré la participation de membres de la fratrie au primaire et au secondaire. Après retranscription des entrevues et codage des données, nous avons procédé à une analyse de contenu thématique (Blanchet et Gotman, 2001). Nous présentons ici les résultats portant sur le point de vue des élèves en utilisant les rubriques suivantes, abordées dans la problématisation précédente et relatives à l'expérience socioscolaire des élèves issus de l'immigration récente: la déclinaison identitaire, le rapport au français et aux matières enseignées, le suivi scolaire par les parents et le projet scolaire et enfin la vie dans le quartier et la communauté. Résultats et discussion sont présentés simultanément pour en faciliter la lecture.

\section{Déclinaison identitaire}

Au tout début des entrevues avec les jeunes, nous avons voulu savoir comment les élèves définissaient leur identité, avec la question la moins prescriptive possible : "Qui es-tu? ». " Je ne sais pas » représente près du quart des réponses à cette question. Ceux qui ont répondu se définissent en fonction de leur âge, leurs goûts, leurs loisirs, leurs qualités et défauts, leur tempérament, l'école qu'ils fréquentent, leur niveau scolaire, etc.

J'aime la couleur bleu, j'aime danser. J'ai 4 sœurs, pas de frère. J'aime pas les cantaloups et les personnes qui sont trop attachantes parce que c'est énervant, mais j'aime les melons d'eau. Je suis deuxième de ma famille. Je suis en $6^{\mathrm{e}}$ année. (M1J4 - 12 ans)

Je suis une fille qui aime faire rire et qui aime faire sortir les choses positives des personnes. Moi, j'adore aider et j'aime donner des conseils. J'adore dessiner [...] Moi, je suis une fille très pacifique et un peu mature. J'aime m'exprimer à ma manière. Je peux dire que je suis un peu pacifique et heureuse. Je suis très positive. (M3J2 - 12 ans)

Je suis un garçon. J'aime jouer à des jeux vidéo, parler avec des amis. On parle, on fait des blagues, on va au cinéma. J'aime les comédies et les films qui font peur. (M9J6 - 14 ans)

Nous étions curieuses de la place de l'identité ethnoculturelle dans la déclinaison identitaire développée par les élèves. Peu de réponses vont dans ce sens. Nous notons que, dans ces réponses, les dimensions relatives à l'ethnicité et à la culture côtoient d'autres marqueurs identitaires.

Je comprends un peu le créole, mais je ne parle pas. Je pense que je parlerai un jour. (M1J5 - 12 ans)

Je viens du Mexique, de Laguna. Je suis né là-bas et après, je suis allé à Juarez et j'ai habité là jusqu'à 8 ans. (M3J6 - 9 ans)

Je suis une enfant de 8 ans, je suis brune et je suis grande de taille. J'ai une peau mélangée avec du brun. (M9J3 - 8 ans)

Nous avons voulu retracer une certaine déclinaison de l'identité ethnoculturelle à travers les réponses aux autres questions de l'entrevue. Interrogés sur " leurs ami(e)s à l'école ", certains élèves parlent explicitement de l'altérité ethnoculturelle. L'animation des entrevues ayant été faite par des personnes différentes, il se peut que le procédé de relance-explicitation des questions sur l'amitié ait produit cet effet constaté. Cependant, même dans ce cas, la forme des réponses et le bref argumentaire sur ce qui fonde l'amitié en question laissent penser que les informations sur l'origine ethnoculturelle des pairs sont accessibles dans le réseau relationnel des élèves et que celle-ci est souvent invoquée de manière complexe, non simpliste. 
II y a des Chinois qui traînent avec des Chinois, mais moi, je suis pas comme ça. [...] Je parle aux Cambodgiens, aux Philippinos... II y a un Latino qui est dans ma classe et il est vraiment super sympa. II y a les Haïtiens, les Jamaïcaines... (M9J8 - 14 ans).

J'ai 3 amies : Ariane vient du Québec, Lisa du Nicaragua et Mélissa du Québec. Elles sont toujours là pour moi, si j’ai besoin d'aide je peux compter sur elles. (M1J3 - 12 ans)

L'analyse du corpus a révélé que les enjeux de définition identitaire sont également présents dans les réponses à une autre question sur «les différents endroits fréquentés/aimés par les élèves dans leur quartier ». La majorité des élèves mentionnent un lieu de culte (église ou mosquée). Nous ne savons pas dans quelle mesure les élèves sont libres ou non de fréquenter ces lieux de culte. Cependant, d'après les développements sur les bénéfices d'une telle fréquentation par les élèves, nous pouvons conclure, de manière prudente, que l'appartenance religieuse semble faire partie de la déclinaison identitaire de ces élèves, quoique selon une importance difficile à cerner.

Comme je suis catholique, j'écoute un peu la parole de Dieu, mais on parle plus de qu'est-ce qu'on a fait aujourd'hui, est-ce qu'on a un problème. On est plusieurs jeunes ensemble et on parle de ça, mais c'est tout en espagnol. J'aime y aller parce que je peux expliquer comment je me sens, si je me sens triste [...] et ils me donnent des conseils. (M3J2 - 12 ans)

\section{Le rapport au français et aux autres matières enseignées}

Questionnés sur ce qu'ils aiment le plus ou le moins apprendre et faire à l'école ou en classe, les élèves ont abordé toutes les matières enseignées, si on se réfère à la présence de ces dernières dans les réponses aux deux types de question. Ainsi, ils nous ont parlé de leur rapport aux mathématiques, à l'éducation physique, à l'histoire, à la géographie, aux sciences, aux arts, etc.

J'aime beaucoup la géographie, l'histoire et les mathématiques, je crois, c'est tout. Mais j'aime mieux l'histoire, j'ai aimé I'histoire sur l'Afrique du Sud, surtout lorsqu'on parle de l'apartheid. (M1J1 - 12 ans)

J'aime les sciences, je ne sais pas tellement pourquoi. Mais en sciences, on fait plein d'expériences. On apprend de nouvelles choses [...]. J'ai aussi appris comment se forme le cerveau du bébé. (M1J5 - 12 ans)

Parce que le français est la langue d'enseignement et la langue officielle du Québec, une langue au cœur des enjeux d'intégration des immigrants, nous avons voulu analyser de près le discours des jeunes à propos du français comme objet d'apprentissage. Grâce à divers mots clés (français, grammaire, écriture, lecture, orthographe, etc., et leurs variantes) appliqués à notre corpus, nous avons constaté qu'une majorité de jeunes se sont prononcés à ce sujet, certains expliquant pourquoi ils aiment apprendre le français et d'autres témoignant des défis relatifs à son apprentissage.

Pour ce qui est des langues les plus utilisées à la maison, tous mentionnent le français ou la combinaison langue maternelle-français. Si nous prenons le cas de l'élève M3J4, il a débuté son expérience scolaire au Maghreb, a fréquenté la classe d'accueil et dit parler arabe et français à la maison. II semble vivre une expérience positive dans son processus d'appropriation de la langue seconde que constitue pour lui le français, à travers différents leviers motivationnels : découvrir (des verbes, des mots), trouver du sens (additionner, chanter), partager un processus d'acculturation linguistique avec un membre de la famille. L'élève M1J7 est quant à elle née en Haïti, y a commencé sa scolarité et n'a pas fréquenté la classe d'accueil au Québec. Son discours fait ressortir d'une certaine manière des enjeux politico-identitaires, mais surtout le statut de langue d'enseignement du français : la maîtriser permet de mieux réussir dans les autres matières.

J'ai appris à conjuguer des verbes... plein de verbes que je ne savais pas quand j'étais en Algérie. J'ai appris à bien parler en français et pas faire des erreurs. J'ai appris à chanter, à additionner... Quand j'apprends beaucoup, après, quand je reviens à la maison, ça va impressionner ma mère : si elle ne connaît pas un verbe, je lui explique c'est quoi. (M3J4 - 10 ans)

[...] le français, parce qu'au Canada on parle français. Je connais beaucoup de choses sur le français. La lecture, [...] les devoirs qu'on donne en classe en mathématiques sont en français. (M1J7 - 10 ans) 
Une minorité d'élèves, au profil hétérogène (génération d'immigration, passage en classe d'accueil, langues parlées à la maison), dit moins aimer apprendre le français. Parmi les extraits transcrits ci-dessous, celui de l'élève M1J3, qui est né hors Canada et qui n'a pas fréquenté la classe d'accueil, met l'accent sur ce qui semble être une difficulté intrinsèque dans l'apprentissage du français : la grammaire. L'élève M9J6, qui est en classe d'accueil, souligne le fait que l'approche pédagogique de l'enseignement du français ne le motive pas. Les élèves M1J5, M9J7 et M9J8 sont tous nés au Canada et font un lien explicite avec le fait que le français n'est pas leur langue maternelle pour expliquer leurs difficultés d'apprentissage.

C'est toujours compliqué en grammaire [...] parce qu'il y a toujours des exceptions partout en grammaire. Puis il faut que tu les apprennes par cœur. (M1J3 - 12 ans)

[...] le cours de français, parce que c'est plate. On fait toujours la même chose et l'enseignant parle toujours. On fait rien, il n'y a rien qui me met au défi. (M9J6 - 14 ans)

[...] le français. Je trouve que c'est une langue très difficile pour moi. La première langue que j'ai pratiquée, depuis que je suis petite, c'est l'anglais. (M9J7 - 13 ans)

Évidemment, les défis dans la maîtrise du français revêtent un caractère spécial pour les élèves qui sont passés par les classes d'accueil. Leur profil est hétérogène tant du point de vue de leur compétence en français que de leur niveau de maîtrise de leur première langue d'apprentissage (qui n'est pas toujours leur langue maternelle) et d'autres matières dans cette langue. Il y a d'abord cette impression, que ressentent des élèves de la classe d'accueil, de perte d'acquis de formation, de « recommencer à zéro ". D'autres conjuguent ensuite ces défis avec la déstabilisation relative à la découverte d'un format de programme plus ou moins différent, déstabilisation qui peut être vécue aussi par des élèves francophones immigrants. Malgré tout, les élèves que nous avons rencontrés trouvent globalement positive l'expérience de la classe d'accueil.

J'ai appris à écrire le français et les mots. Maintenant, j'ai encore beaucoup de problèmes parce que je ne parle pas à la maison. Je commence à oublier les homophones. (M3J6 - 9 ans)

J'apprends à lire et à écrire, les verbes et les maths en français parce que là-bas, c'est pas la même chose qu'ici. Quand tu fais les divisions, ce n'est pas la même façon de faire... faire des additions et des divisions; je ne sais pas, c'était différent. (M3J7 - 9 ans)

Oui, j'étais à la classe d'accueil; ils disaient que je ne savais pas bien lire. Ça allait bien, mais je ne savais pas lire. Je n'ai pas beaucoup aimé ça parce qu'elle [sa professeure] criait sur moi. (M9J2 - 8 ans)

Au moment des entrevues, la majorité des jeunes, comme M1J3, pensent que leur année scolaire est en voie d'être réussie. Ils se basent sur des constats de bons résultats scolaires, d'amélioration et d'attitudes persévérantes de leur part. Parmi ceux qui pensent avoir moins bien réussi leur année scolaire, comme M1J8, la plupart des difficultés mentionnées sont en lien avec l'apprentissage du français. M1J3 et M1J8 sont respectivement de première et de deuxième génération, partageant le même pays d'origine.

Oui. [...] J'ai obtenu des bonnes notes à mes examens, j'ai travaillé fort pour les obtenir aussi. [...] J'ai étudié tout ce qu'on me disait d'étudier, j'ai fait tous mes devoirs sans exception; puis, à chaque fois que le prof donnait des explications, j'écoutais. (M1J3 - 12 ans)

Il y a des choses que je devais savoir que je ne savais pas encore l'année passée. Je devrais faire plus d'études. Je commence à apprendre là, mais avant, je ne savais pas bien les divisions, je ne savais pas bien mes tables de multiplication. Je ne sais pas c'est quoi en français, un synonyme et un antonyme. À cause de ces choses, même si je suis en $6^{\mathrm{e}}$ année, je ne considère pas que j'ai réussi mon année. (M1J8 - 12 ans) 


\section{Le suivi scolaire par les parents et le projet scolaire}

Selon la presque totalité des jeunes, les parents s'intéressent explicitement à leur vécu socioscolaire et s'enquièrent d'aspects divers : apprentissage, sentiment de bien-être à l'école, relations avec les amis ou pairs, incidents vécus, etc. Même pour les deux élèves qui semblent faire exception, l'une mentionne que sa mère, étant bénévole à l'école, a accès à beaucoup d'information concernant sa fille. L'autre élève précise qu'elle aime prendre les devants et partager ce qu'elle vit à l'école, anticipant ainsi les questions de ses parents. La majorité des parents consultent l'agenda scolaire de leur enfant, surtout pour prendre connaissance des messages des enseignants.

Pour la réalisation des devoirs, certains disent recevoir de l'aide de leurs parents, non pas sur une base régulière, mais quand c'est vraiment nécessaire. Des jeunes ont souligné la barrière de la langue pour justifier le peu d'aide reçue de la part des parents pour la réalisation des devoirs. D'autres disent que les ressources familiales en matière d'aide aux devoirs et de suivi de l'agenda sont aussi à chercher auprès d'autres membres de la famille (frère, sœur, oncle, grands-parents).

Ils ne vérifient pas toujours si j'ai des devoirs [...]. Quand j'ai besoin d'aide je demande à mon père, parce que ma mère ne parle pas beaucoup le français. Mon père comprend plus bien le français que ma mère. (M1J4 - 12 ans)

Comme c'est Mamie qui est là le plus souvent, c'est elle qui regarde mon agenda. Même quand mon père est là, des fois, c'est Mamie qui le fait toujours, mais des fois c'est Papi qui le fait comme ma mère. (M1J1 - 12 ans)

Selon plusieurs jeunes, dans la communication avec l'école, leurs parents préfèrent la médiation de l'agenda et du téléphone au déplacement à l'école. Ils s'y rendent rarement sans y avoir été convoqués. Cependant, presque tous les élèves que nous avons rencontrés jugent que leurs parents devraient aller à l'école plus souvent pour s'informer du vécu socioscolaire de leur enfant et pour savoir comment mieux soutenir leur réussite à la maison.

Oui. Comme ça, ma mère sait qu'est-ce que j'ai du mal à faire et c'est ça qu'on va travailler. (M3J1 - 12 ans)

Si l'enfant a des difficultés, c'est important. Si l'enfant a des bonnes notes, je trouve pas... (M9J5 - 16 ans)

Nous avons aussi voulu savoir comment les élèves rencontrés voient leur projet scolaire. Lorsque nous avons demandé aux jeunes s'ils savent ce que leurs parents pensent de l'école, ils disent avoir perçu chez eux une appréciation globalement positive :

Ils pensent que c'est mieux ici qu'au Mexique. (M3J6 - 9 ans)

Ils trouvent ça très important; ils disent que c'est bon pour nous et que ça va nous rapporter gros, plus tard. (M9J11 12 ans).

Questionnés à propos des motivations qui les poussent à venir à l'école et des perspectives d'avenir qu'ils entrevoient à l'issue de leurs études, les élèves ont exposé, dans leurs mots, des éléments relatifs à la plus-value de l'école et aux possibilités que la qualification scolaire ouvre. Selon eux, la fréquentation scolaire permet l'accès aux savoirs-compétences-connaissances, le développement personnel, l'autonomie, la socialisation à de nouveaux univers et les moyens d'une mobilité sociale.

Je vais à l'école parce que je veux être intelligent; je ne veux pas être fou. Je veux être une grande personne et avoir une famille et un bon travail. [...] Si je viens pas à l'école, je n'aurai rien. (M3J1 - 12 ans)

Mais je vais à l'école pour apprendre à lire, à écrire, pour savoir comment calculer. Si je vais pas à l'école, plus tard, je vais pouvoir rien faire comme travail, je vais pas pouvoir gagner ma vie. Par exemple, si je suis plus bonne en français, sûrement que je vais faire professeur ou avocate, des trucs où on parle plus, où on est plus sur le français. (M1J9 - 11 ans)

Pour apprendre de nouvelles choses puis pour avoir des amis. C'est ce qui me motive pour aller à l'école. [...] Ben on va à l'école pour avoir de nouvelles expériences avec les élèves, les enseignants, pour apprendre de nouvelles choses, de nouvelles matières. Puis aussi pour se détacher un peu des parents. (M1J3 - 12 ans) 
Pour ce qui est de leurs aspirations professionnelles, les métiers de la santé sont les plus souvent nommés (notamment médecine et soins infirmiers) par les élèves, suivis par le journalisme et l'enseignement. II y a par la suite mention d'une variété d'autres métiers: coiffure, arts, restauration, mannequinat, services de sécuritéincendie, police, etc. Les modèles ou idoles qui les inspirent sont des personnalités publiques (acteurs, chanteurs, etc.), des personnages fictifs (super-héros, personnages de séries télévisées, etc.), des membres de leur famille (père ou mère, tante ou oncle, etc.) et de leur entourage.

Lors de l'entrevue, nous avons posé la question suivante aux élèves : "Est-ce que tu parles à tes parents de ce que tu voudrais faire plus tard? Sont-ils d'accord avec toi? »Plusieurs disent que le sujet a été abordé et que dans l'ensemble les parents respectaient leurs choix, tout en y allant de suggestions pour les orienter. Et le discours des jeunes semble révéler les enjeux de l'imbrication entre projet scolaire des enfants et projet migratoire familial, mais aussi des stratégies des jeunes pour atténuer la pression de cette imbrication, ou pour la définir autrement.

À ma mère et à mon père, oui, à la famille en général, des fois j'en parle. Ma mère est professeure de sciences, je lui parle souvent de mes expériences à l'école. Mes parents sont ouverts à cela. Le soir à la maison on parle de ce qu'on veut faire plus tard, ils écoutent. C'est sûr que je suis venue pour mes études ici, ils m'ont dit que je dois me concentrer sur l'école. J'ai pas envie être professeure comme ma mère, je n'ai pas envie être ingénieure comme mon père. (M1J1 - 12 ans)

À chaque métier, ils vont te donner des trucs mauvais et ça te décourage de continuer d'avoir des recherches dessus. Moi, je fais tout moi-même et je ne veux pas les mettre dedans. Je vais peut-être en parler à mes cousins qui sont plus vieux que moi et qui ont plus d'expérience. (M9J8 - 14 ans)

Finalement, nous avons cherché à savoir si ces jeunes avaient le sentiment qu'il serait aisé pour eux de faire ce qu'ils souhaitent plus tard. Dans l'ensemble, ils ont répondu avec une certaine confiance en leurs capacités de réaliser leurs rêves. Toutefois, la plupart d'entre eux se disent conscients du fait qu'ils devront travailler très fort pour atteindre leurs buts. Au-delà de l'auto-imputabilité quant aux efforts à faire pour réussir, certains abordent plus ou moins explicitement la perception de facteurs contextuels qui pourraient entraver leur réussite.

Oui, quand même. Si j'étudie et je fais des efforts, j'y arriverai. (M3J1 - 12 ans)

Je ne sais pas vraiment, ma tante, elle a fait des études au Cameroun, mais elle n'a pas de travail maintenant. C'est sûr qu'elle voulait travailler dans sa vie, mais elle n'a pas trouvé de travail. Bon, ça peut être pareil pour moi. (M1J1 - 12 ans)

\section{Le jeune et son quartier}

La dernière section de l'entrevue avec les élèves concerne leur vie de quartier et les activités qu'ils y pratiquent, les endroits où ils vont en dehors de la maison et de l'école et les raisons pour lesquelles ils les fréquentent. Plusieurs d'entre eux ont parlé de la fréquentation d'un lieu de culte, et les motifs évoqués sont les suivants : la prière, l'apprentissage de la religion, différentes activités cultuelles, la rencontre de gens, la participation à des activités ludiques. La grande majorité des jeunes ont raconté comment ils naviguent à travers les différents espaces de leur quartier (parc, bibliothèque, centre d'achat, etc.).

J'aime bien ce quartier, il y a beaucoup de choses tout près. Il y a une bibliothèque juste à côté, un parc, une piscine et une école. Quand je vais au parc, je joue dans les manèges ou j'écoute de la musique. Quand je vais à la bibliothèque, je loue des livres ou je lis. Quand je vais à la piscine, je reste dans l'eau et je me baigne avec mes amies et mes cousines. (M1J1 - 12 ans)

Nous avons posé plusieurs questions aux jeunes concernant leur participation à des activités spécifiques offertes par des organismes communautaires situés dans leur quartier, en collaboration avec les écoles. Les activités sont diversifiées : arts, activités sportives, sorties, camps d'été, aide aux devoirs, etc. Plusieurs jeunes pensent que leur participation aux activités organisées par les organismes communautaires les aide à améliorer leur expérience scolaire: certaines sorties éducatives permettent d'acquérir de nouvelles connaissances et d'autres, d'élargir leurs horizons vers de nouveaux divertissements. Des élèves prétendent qu'ils aiment fréquenter ces activités parce qu'ils y font la rencontre d'autres jeunes de leur âge, qu'ils apprennent à collaborer, à travailler en équipe, à avoir une meilleure confiance en soi, à gérer des conflits interpersonnels, etc. Une élève récemment immigrée a affirmé 
que sa participation au camp d'été l’a aidée à mieux connaître son nouvel environnement et à savoir quelles activités faire à Montréal.

On est allés à la piscine, au parc, au Biodôme et à l'Insectarium. On est allés à beaucoup d'endroits... On est allés au VieuxPort, au cinéma, au labyrinthe. (M3J1 - 12 ans)

$\mathrm{Si}$, par exemple, à XXX [organisme communautaire] on va visiter le Biodôme, on apprend d'autres espèces d'animaux, on connaît plus de trucs, ça aide aussi à l'école, oui. Ce qu'on fait là complète le travail de l'école. (M1J1 - 12 ans)

Donc les jeunes semblent apprécier un certain niveau de collaboration entre leur école et les organismes communautaires. L'approche différenciée entre les deux espaces de socialisation paraît généralement leur convenir, notamment le fait de profiter de la supervision d'adultes (acteurs scolaires et intervenants communautaires) aux mandats différents mais complémentaires. De manière schématique, la majorité d'entre eux considèrent que l'école est surtout un espace d'apprentissage structuré, " on apprend en travaillant ", tandis que les activités à l'organisme communautaire permettent de s'amuser dans un cadre où les balises sont plus flexibles, « on apprend en s'amusant».

\section{Conclusion}

Revenons sur les principaux résultats présentés dans cet article. Les élèves que nous avons rencontrés ont partagé leur point de vue sur leur déclinaison identitaire, leur rapport aux apprentissages, leur projet scolaire et leur utilisation de différents espaces et ressources de leur quartier. Tout d'abord, les données révèlent la complexité de l'auto-identification ethnoculturelle de la part des élèves. Selon Amin, Poussin et Martinez (2008), la prévalence de l'auto-identification ethnoculturelle varie d'un groupe à l'autre, dépendant notamment des rapports intergroupes et du sentiment d'exclusion ressenti. Par exemple, dans leur recherche, les jeunes Français originaires du Maghreb y ont plus recours que ceux originaires de Turquie. Dans le cas des élèves que nous avons rencontrés, nous pensons que leur jeune âge pourrait aussi induire une certaine pudeur à se définir directement soi-même par son origine ethnoculturelle. Il est aussi possible que la conscience, chez certains, de l'existence d'un potentiel de stigmatisation de leur identité ethnoculturelle les rende réticents à en parler. Dans tous les cas, nous n'avons pas senti de posture de surenchère identitaire dans ce processus, mais avons décelé une certaine appropriation de signifiants relatifs à la langue maternelle et à la religion. De plus, l'allusion significative à l'identité ethnoculturelle des pairs dans l'explicitation des liens d'amitié révèle un réseau relationnel pluriethnique. Nous pensons donc que, de la même façon qu'une "décrispation de la culture sociale et politique» (Lorcerie, 2003, p. 272) est bénéfique au niveau de la société, les acteurs scolaires devraient encourager une mixité socioculturelle dans les interactions sociales entre élèves (Duong et Kanouté, 2007). Nos données montrent que les défis relatifs à l'appropriation du français et à l'identification à sa langue maternelle concernent à la fois des élèves immigrants de première et de deuxième générations. Les témoignages des élèves semblent indiquer que le milieu scolaire ne tient pas compte suffisamment de leur langue maternelle ou de leur langue d'usage autre que le français pour mieux les aider à s'approprier ce dernier. Nous partageons donc l'avis de chercheurs qui plaident pour une incorporation positive du plurilinguisme à l'école, sans que cela soit vécu comme une menace pour le vivre-ensemble ou pour la vitalité du français en tant que langue d'enseignement (Armand et Dagenais, 2012).

Est-il possible d'atténuer les effets combinés de la conjugaison entre défavorisation et immigration récente? L'école peut constituer un tuteur de résilience (Cefaï, 2007) dans un contexte de défavorisation, en travaillant sur l'accueil, la convivialité, la reconnaissance du milieu et de ses ressources, l'efficience des dispositifs didactiques et pédagogiques, notamment l'accompagnement de la construction du projet scolaire de l'élève et de son arrimage à un projet de vie (Delannoy, 2005; Kanouté, 2007b; René et Laurin, 2009). Par exemple, le fait de donner des devoirs à faire à domicile est une pratique emblématique de la culture de l'école, et les acteurs scolaires n'ont que récemment commencé à se questionner sérieusement sur les «enjeux cognitifs et sociaux d'une pratique ordinaire " (Rayou, 2010), qui met à l'épreuve le capital humain et socioculturel familial, notamment lorsque les parents ne maîtrisent pas suffisamment le français ou ont de la difficulté à décoder la culture et les pratiques scolaires. Nos données montrent aussi que ce n'est pas parce que les parents immigrants sont moins présents à l'école qu'ils se désinvestissent du suivi scolaire (Kanouté et coll., 2008). Encore une fois, la contribution des organismes communautaires à l'épanouissement du jeune et de l'élève est manifeste: il reste à peaufiner 
l'apprivoisement réciproque des acteurs scolaires et des intervenants communautaires pour le bien-être des jeunes et de leurs familles. Les jeunes que nous avons rencontrés ont partagé les bénéfices qu'ils tirent de la décentralisation des activités d'apprentissage à d'autres espaces extrascolaires, notamment les occasions de mieux connaître la société d'accueil et d'intensifier ainsi le processus d'acculturation. Ils ont mentionné les retombées positives de la fréquentation d'espaces communautaires à des fins de loisirs, de soutien aux apprentissages scolaires et pour des raisons culturelles et religieuses. Il nous semble que ces résultats confirment, une fois de plus, la nécessité que les écoles deviennent proactives dans la coordination d'une synergie communautaire pour le bienêtre global des élèves (Kanouté et coll., 2016).

Pour terminer, le discours des élèves rencontrés montre encore une fois qu'une perspective interculturelle à l'école devrait se penser en lien étroit avec l'expérience socioscolaire de l'élève (Kanouté, 2007b) et, minimalement, reconnaître l'élève dans ses identités et ses acquis d'apprentissage, insuffler un certain hétérocentrisme dans l'interprétation du programme, expliciter sans ostracisme le "vivre-ensemble " qui demande à ce que l'école socialise tous les élèves, gérer le quotidien scolaire de manière inclusive et enfin prendre l'initiative de développer un partenariat avec les familles et la communauté. II nous semble évident aussi que la perspective interculturelle à l'école, au-delà de cibler le confort identitaire des élèves dans ce milieu de vie, ne doit pas perdre de vue l'égalité des chances de réussite scolaire. L'échec, quelles que soient les causes qu'on lui attribue, joue un rôle important dans le cercle vicieux de la stigmatisation-exclusion.

\section{Références bibliographiques}

Abou, S. (2002). L'identité culturelle suivi de Cultures et droits de l'homme. Perrin et Presses de l'Université SaintJoseph.

Amin, A. (2012). Stratégies identitaires et stratégies d'acculturation : deux modèles complémentaires. Alterstice, 2(2), 103-116.

Amin, A., Poussin, M. et Martinez, F. (2008). Le rôle du sentiment d'exclusion et des perceptions de la société dans le processus d'identification chez les jeunes français issus de l'immigration. Les Cahiers Internationaux de Psychologie Sociale, 80, 27-38.

Armand, F. et Dagenais, D. (2012). S'ouvrir à la langue de l'Autre et à la diversité linguistique. Revue de l'Association canadienne d'éducation (ACE), 52(1).

Armand, F., Rousseau C., Lory, M.-P. et Machouf, A. (2011). Les ateliers d'expression théâtrale plurilingue en classe d'accueil. Dans F. Kanouté et G. Lafortune (dir.), Familles d'origine immigrante au Québec : enjeux sociaux, de santé et d'éducation (p. 97-111). Montréal : Presses de l'Université de Montréal.

Berry, J. (2005). Acculturation: Living successfully in two cultures. International Journal of Intercultural Relations, 29, 697-712.

Blanchet, A. et Gotman, A. (2001). L'enquête et ses méthodes : l'entretien. Paris : Nathan.

Boudarbat, B. et Grenier, G. (2014). L'impact de l'immigration sur la dynamique économique du Québec. Rapport remis au ministère de l'Immigration, de la Diversité et de l'Inclusion.

Cefaï, C. (2007). Resilience for All: A study of classrooms as protective contexts. Emotional and Behavioral Difficulties, 12(2), 199-134.

Chen, C., Smith, P. et Mustard, C. (2010). The prevalence of over-qualification and its association with health status among occupationally active new immigrants to Canada. Ethnicity and Health, 15(6), 601-619.

Cohen, E. (1994). Le travail de groupe : stratégies d'enseignement pour la classe hétérogène. Traduit par F. Ouellet. Montréal : Chenelière.

Comité de gestion de la taxe scolaire de l'lle de Montréal (CGTSIM) (2015). Portrait socioculturel des élèves inscrits dans les écoles publiques de l'île de Montréal. Inscriptions au 14 novembre 2014. Montréal : CGTSIM. http://www.cgtsim.qc.ca/fr/documents-site-web/129-portrait-socioculturel-2015/file 
Conseil national de l'évaluation du système scolaire de la France (CNESCO) et Conseil supérieur de l'éducation du Québec (CSE). (2015). La mixité sociale à l'école. Québec : Bibliothèques et Archives nationales du Québec.

Dansereau, F. et Germain, A. (2002). Fin ou renaissance des quartiers? Les significations des territoires de proximité dans une ville pluriethnique. Espaces et Sociétés, 108-109, 7-28.

Delannoy, C. (2005). La motivation : désir de savoir, décision d'apprendre. Paris : Hachette.

Duong, L. et Kanouté, F. (2007). Les interactions sociales de l'élève immigrant à Montréal. Revue de l'Université de Moncton, 38(2), 73-105.

Glennerster H. (2002). United States poverty studies and poverty measurement: The past twenty-five years. Social Science Review, Mars, 83-107.

Hohl, J. (1996). Qui sont les " parents »? Le rapport de parents immigrants analphabètes à l'école. Lien social et Politiques, 35.

Kanouté, F. (2002). Profils d'acculturation d'élèves issus de l'immigration récente à Montréal. Revue des sciences de l'éducation, 28(1), 171-190.

Kanouté, F. (2007a). Les parents et leur rapport à l'école : une recherche en milieu défavorisé. Dans C. Solar et F. Kanouté (dir.), Équité en éducation et formation (p. 25-46). Montréal : Éditions Nouvelles.

Kanouté, F. (2007b). La pratique de l'interculturel. Dans C. Solar et F. Kanouté (dir.), Équité en éducation et formation (p. 121-140). Montréal : Éditions Nouvelles.

Kanouté, F., Charette, J., André, J., Rachédi, L. et Rahm, J. (2014). Dynamiques d'établissement, enjeux scolaires et ressources communautaires : point de vue de parents d'origine haïtienne et d'intervenants. Revue Diversité urbaine, 14(2), 31-50.

Kanouté, F. et Lafortune, G. (2011). Familles québécoises d'origine immigrante : les dynamiques d'établissement. Montréal : Presses de l'Université de Montréal.

Kanouté, F. et Lafortune, G. (2014). L'intégration des familles d'origine immigrante : les enjeux sociosanitaires et scolaires. Montréal : Presses de l'Université de Montréal.

Kanouté, F., Lavoie, A., Guennouni, R. et Charette, J. (2016). Points de vue d'acteurs scolaires et d'intervenants communautaires sur les besoins d'élèves immigrants et de leur famille dans des écoles défavorisées à Montréal. Revista Interuniveritaria de Formacion del Profesorado, 53, 19(1), 141-155.

Kanouté, F., Vatz Laaroussi, M., Rachédi, L. et Tchimou Doffouchi, M. (2008). Familles et réussite scolaire d'élèves immigrants du secondaire. Revue des sciences de l'éducation, 34(2), 265-289.

Lafortune, G. et Kanouté, F. (2007). Vécu identitaire d'élèves de $1^{\mathrm{e}}$ et de $2^{\mathrm{e}}$ génération d'origine haïtienne. Revue de I'Université de Moncton, 38(2), 33-71.

Landes, X. (2011). La dimension numérique dans le concept de minorité : efficience et justice ethnoculturelle. Les ateliers de l'éthique / The Ethics Forum, 6(2), 49-78.

Lareau, A. (2003). Unequal childhoods. Class, race, and family life. Berkeley/Los Angeles/London : University of California Press.

Lorcerie, F. (2003). L'école et le défi ethnique. Paris : ESF.

Martiniello, M. (1995). L'ethnicité dans les sciences sociales Paris : Presses universitaires de France.

Meintel, D. (1995). L'identité ethnique chez de jeunes Montréalais d'origine immigrée. Sociologie et Sociétés, 24(2), 73-89.

Millet, M. et Thin, D. (2005). Le temps des familles populaires à l'épreuve de la précarité. Lien social et Politiques, (54), 153-162.

Alterstice - Revue Internationale de la Recherche Interculturelle, vol. 6, $n^{\circ} 1$ 
Ministère de l'Immigration, de la Diversité et de l'Inclusion (MIDI). (2015). Présence en 2015 des immigrants admis au Québec de 2004 à 2013. Québec : MIDI. http://www.midi.gouv.qc.ca/publications/fr/recherchesstatistiques/PUB_Presence2015_admisQc.pdf

Ninacs, W. (2008). Empowerment et intervention : Développement de la capacité d'agir et de la solidarité. Québec : Presses de l'Université Laval.

Papazian-Zohrabian, G. (2013). Le deuil traumatique chez l'enfant et son influence sur la construction de son identité. Revue québécoise de psychologie, 34(2), 83-100.

Rayou, P. (2010). Faire ses devoirs: Enjeux cognitifs et sociaux d'une pratique ordinaire. Rennes : Presses universitaires de Rennes.

Rahm, J. (2007). Learning and becoming across time and space: A look at learning trajectories within and across two inner-city youth community science programs. Dans W.-M. Roth et K. Tobin (dir.), Science, learning, and identity : Sociocultural and cultural-historical perspectives (p. 63-79). Rotterdam : Sense Publisher.

René, J.-F. et Laurin, I. (2009). Transmettre la parole de parents en milieu de pauvreté : quand le chercheur devient médiateur. Nouvelles pratiques sociales, 21(2), 60-76.

Vallet, L.-A. (2005). La mesure des effets de quartier/voisinage: un objet important et difficile à la croisée des sciences sociales. Revue économique, 56(2), 362-370.

Vatz Laaroussi, M. (1996). Les nouveaux partenariats famille-école au Québec : I'extériorité comme stratégie de survie des familles défavorisées ? Lien social et Politiques, 35, 87-97.

Vatz Laaroussi, M., Kanouté, F. et Rachédi, L. (2008). Les divers modèles de collaborations familles immigrantesécoles : de l'implication assignée au partenariat. Revue des sciences de l'éducation, 34(2), 291-311.

Yin, R. (2003). Case study research. Design and method ( $3^{\mathrm{e}}$ éd.). Thousand Oak, London, New Delhi: Sage Publications. 